This article has been published in a revised form in Behavioural and Cognitive Psychotherapy https://doi.org/10.1017/S135246581600028X. This version is free to view and download for private research and study only. Not for re-distribution or re-use. (C) the authors.

\title{
What IAPT CBT High-Intensity Trainees Do After Training
}

\author{
Sheena Liness \\ Institute of Psychiatry, Psychology and Neuroscience, King's College London, UK \\ Susan Lea \\ University of Greenwich, London, $U K$ \\ Steffen Nestler and Hannah Parker \\ Institute of Psychiatry, Psychology and Neuroscience, King's College London, UK \\ David M. Clark \\ University of Oxford, UK
}

Correspondence to Sheena Liness, Department of Psychology, Institute of Psychiatry,

Psychology and Neuroscience, King's College London, 16 De Crespigny Park, Denmark

Hill, London SE5 8AF, UK. E-mail: sheena.liness@kcl.ac.uk 
Background: The UK Department of Health Improving Access to Psychological Therapies (IAPT) initiative set out to train a large number of therapists in cognitive behaviour therapies (CBT) for depression and anxiety disorders. Little is currently known about the retention of IAPT CBT trainees, or the use of CBT skills acquired on the course in the workplace after training has finished.

Aims: This study set out to conduct a follow-up survey of past CBT trainees on the IAPT High Intensity CBT Course at the Institute of Psychiatry, Psychology and Neuroscience (IoPPN), King's College London (KCL), one of the largest IAPT High Intensity courses in the UK.

Method: Past trainees $(n=212)$ across 6 cohorts (2008-2014 intakes) were contacted and invited to participate in a follow-up survey. A response rate of $92.5 \%(n=196)$ was achieved. Results: The vast majority of IAPT trainees continue to work in IAPT services posttraining (79\%) and to practise CBT as their main therapy modality (94\%); 61\% have become CBT supervisors. A minority (23\%) have progressed to other senior roles in the services. Shortcomings are reported in the use of out-of-office CBT interventions, the use of disorderspecific outcome measures and therapy recordings to inform therapy and supervision.

Conclusions: Past trainees stay working in IAPT services and continue to use CBT methods taught on the course. Some NICE recommended treatment procedures that are likely to facilitate patients' recovery are not being routinely implemented across IAPT services. The results have implications for the continued roll out of the IAPT programme, and other future large scale training initiatives.

Keywords: CBT training, CBT, follow-up, CBT supervision

\section{Introduction}

The Improving Access to Psychological Therapies (IAPT) initiative has attracted national and international interest. It was set up to train and establish a new workforce to deliver evidencebased treatment in psychological therapy services across England (www.iapt.nhs.uk) based on NICE (National Institute for Health and Clinical Excellence) guidance (www.nice.org.uk). Services follow a stepped care model with High Intensity (HI) therapists offering individual face-to-face therapy (step 3), and Psychological Well-being Practitioners (PWPs) delivering 
low-intensity interventions such as guided self-help (step 2). Training is provided by local Higher Education Institutions (HEIs), and evidence-based therapy for depression and anxiety disorders is delivered in local IAPT services. Although IAPT supports five different HI therapies (CBT, interpersonal psychotherapy, counselling, couples therapy, and brief psychodynamic therapy), CBT accounts for the largest number of HI trainees as it has the broadest indication in current NICE guidelines. The IAPT programme has trained 5745 high (step 3) and low (step 2) intensity CBT workers and 1410 therapists were trained in the other NICE-recommended modalities (IAPT Programme NHS England, 2015). Large scale training is only effective if trainees stay working in services posttraining and continue to use the skills learned. This paper looks at this issue for High Intensity CBT trainees.

Follow-up surveys of multi-professional postgraduate CBT training courses are small in number, and none have focused on IAPT trainees. Ashworth, Williams and Blackburn (1999), Kennedy-Merrick, Haarhoff, Stenhouse, Merrick and Kazantzis (2008), and MacLiam (2015) achieved an average response rate of 67\% (56-80\%), reported 34\% (24$44 \%$ ) of respondents as specifically employed as CBT therapists, and 71\% (51-90\%) having CBT as a main focus of their clinical work posttraining. Follow-up surveys of nurse behaviour therapy training courses (Brooker and Brown, 1986; Gournay, Denford, Parr and Newell, 2000; Newell and Gournay, 1994; Ryan, Cullinan and Quayle, 2005) had an average response rate of $66 \%(53-80 \%)$, with $59 \%$ of respondents employed as nurse therapists posttraining (Newell and Gournay, 1994). Ryan et al. (2005) listed the nurses' primary focus on $\mathrm{CBT}$ as $17 \%$ of their sample. Follow-up surveys of Psycho-Social Interventions (PSI) training courses showed response rates of, on average, $82 \%$ (70-94\%) and reported former trainees using PSI techniques with 56\% (36-69\%) of clients (Brooker, Saul, Robinson, King and Dudley, 2003; Fadden, 1997; Kavanagh et al., 1993). Studies exploring factors that promote or inhibit transfer of CBT training into clinical practice (Kjøge, Turtumoygard, 
Berge and Ogden, 2015; Herschell, Kolko, Baumann and Davis, 2010; Milne, Gorenski, Westerman, Leek and Keengan, 2000) highlight the role of workplace obstacles such as inadequate time and support, in maintaining skills and delivering CBT therapy. Being aware of these limitations, the IAPT initiative was set up to deliver training in a novel way.

Unique to this initiative is a national directive with key documents outlining a national curriculum for CBT training institutions (Department of Health, 2011), and a framework for IAPT services (National IAPT Implementation plan, Department of Health, 2008), which sets out a directive for close working relations between the IAPT training institutions (HEIs) and the IAPT services and workforce. Trainees are employed on a 1-year training contract attending university 2 days and working in IAPT services 3 days a week, delivering therapy to patients they are trained to treat. The IAPT CBT national curriculum (Department of Health, 2011) is adhered to with a mix of workshops and lectures (50\%), experiential learning and skills training (50\%), plus weekly CBT clinical supervision. The CBT training is fully accredited by the British Association for Behavioural and Cognitive Psychotherapies (BABCP). On course completion, graduates are eligible to apply for provisional $\mathrm{BABCP}$ accreditation as a CBT practitioner. The IAPT training institutions (HEIs) and IAPT services work closely together. Posttraining, trainees are employed full time in IAPT services. The time, organization, and cost devoted to this initiative make a follow-up of trainees essential. It is also hoped that this bespoke implementation model is associated with better retention of trainees and reported use of CBT skills posttraining.

\footnotetext{
Aims

The aim of this study was to follow-up graduates of the IoPPN IAPT Post-Graduate Diploma in CBT from the start of the IAPT Programme in 2008 to: (1) examine IAPT workforce retention and career progression; (2) describe graduates' current CBT clinical and supervision
} 
practice, in particular to see if IAPT Course practices/standards, in line with the IAPT National Curriculum (Department of Health, 2011), had been maintained; and (3) to gather feedback from the graduates on their current IAPT work experiences.

\section{Method}

IAPT graduates who completed the CBT Post Graduate training at the IoPPN/KCL from 2008 to 2014 (years 1 to $6, n=225$ ) were invited via email to participate in a follow-up survey. Fails, withdrawals, and drop-outs from the course $(n=13)$ were excluded, leaving 212 eligible to follow-up. Those who agreed to participate were sent a link to consent and complete the electronic survey on Survey Monkey (www.surveymonkey.com). The survey questions were based on previous large-scale follow-up CBT training surveys (Ashworth et al., 1999; Gournay et al., 2000; Newell and Gournay, 1994) and consultation with the CBT Course Team. A questionnaire was created that assessed: demographics and background information, current clinical work and clinical activity, supervision received and provided, as well as therapy competence and work satisfaction. The CBT Competence Framework (Roth and Pilling, 2008) informed the CBT specific techniques included. A combination of open, closed and 5-point Likert scale questions was used to assess how often participants reported using various techniques in posttraining therapy sessions: None (0\%), Few (25\%), Some (50\%), Most (75\%), and Every (100\%).

Each section had space for open-ended responses encouraging further information. The survey was piloted on past trainees and current course supervisors $(n=15)$ and amended in line with feedback. It was not tested for reliability. (A copy is available from the first author on request.)

\section{Ethical considerations}


Ethical approval was granted through King's College London University.

\section{Data analysis}

A range of descriptive parametric statistical procedures were conducted, as well as a Chisquare test on workforce retention. Open-ended responses were entered into Excel and categorized into key themes by the first author. A second CBT therapist independently rated $10 \%$ of the responses to relevant themes. The interrater agreement on each theme varied between moderate (Cohen's $\kappa=0.54)$ and substantial (Cohen's $\kappa=0.69$ ).

\section{Results}

\section{Respondents}

In total, 198 of the 212 eligible trainees from the 6 cohorts (2008-2014) agreed to participate. Two former trainees did not fill in any questions and were excluded from the analysis. This resulted in an overall response rate of $92.5 \%$ (range $83 \%-100 \%$ per year). Details of respondents' age, gender, undergraduate degree, core profession, ethnicity and decade of qualification in core profession are provided in Table 1 . The responder and non-responder groups shared similar demographics.

\section{[Insert Table 1 about here]}

\section{Workforce retention}

At the time of survey completion $79 \%$ of trainees $(n=155)$ were still working in an IAPT service. This rate is reasonably consistent across the six cohorts (2008-2014), with retention between $68 \%$ and $86 \%$ depending on the training year. Interestingly, there is no evidence of a decline in numbers related to time elapsed since course completion (Figure 1). 
[Insert Figure 1 about here]

The percentage of trainees who had stayed in their training IAPT service was $75.5 \%$ ( $n=117)$, and $23.9 \%(n=37)$ had switched to a different IAPT service. The 41 trainees who reported no longer to be working in an IAPT service had stayed in their respective IAPT services for an average of 2.1 years (range: 1-4 years, $S D$ 1.2) before moving on. Clinical psychologists were more likely to move on (27 of 71, exp. count 17.6) compared to CBT therapists who had previously worked as PWPs (5 of 58, exp. count 14.4) (Fisher's exact $P=$ $14.7, p=.0001)$. The primary positions that the trainees switched to after working in IAPT were specialist CBT or general clinical psychologist roles in which they are still doing CBT. There was a significant difference in age between former trainees who were still working in IAPT $($ mean $=37.5$ years, $S D=7.36, n=155)$ and those that had left IAPT (mean $=33.9$ years, $S D 3.49, n=41), t(194)=3.07, p<.001$, indicating a trend for older trainees to stay longer.

CBT remained the main therapy modality for $94 \%$ of participants $(n=184)$ with only $4 \%$ (8) using primarily other forms of therapy and 2\% (4) no longer working as therapists. Most participants still worked in Greater London $(88 \%, n=173)$, while $11 \%$ (17) had moved out of London and 2\% (4) abroad.

\section{Career progression}

On successfully completing the course, trainees are eligible to apply for provisional accreditation as a CBT therapist with the BABCP, followed by full accreditation after 1 year posttraining. A large number of trainees had obtained provisional or full BABCP Therapist Accreditation (Year 1: 82.5\%, $n=33$; Year 2: 79.5\%, $n=35$; Year 3: 71.5\%, $n=20$; Year 4: 
$80 \%, n=20$; Year 5: 42.4\%, $n=14$; Year 6: 30.8\%, $n=8)$ while 28\% (54) of trainees' applications were still in progress, which is to be expected for years 5 and 6. Salary in IAPT services is largely based on the NHS Agenda for Change Salary Bands (NHS, 2015). The majority of trainees were employed at Band 6 during training, and most had progressed to a higher pay band posttraining with $67 \%(n=132)$ at Band 7 and $18 \%(n=35)$ at Band $8 \mathrm{a}$, in line with national expectations. Four had attained $8 \mathrm{~b}(2 \%)$ and one an 8c IAPT Clinical Lead position $(0.5 \%)$.

Clinical work post-training

[Insert Table 2 about here]

The majority of the past trainees' work time was spent in clinical work doing CBT $(76 \%, S D$ 22.1) (Table 2). Other duties were providing clinical supervision (10\%, SD 10.0), managerial/administrative duties $(6 \%, S D$ 10.7), teaching/training $(4 \%, S D$ 8.7), research (2\%, SD 3.9), and miscellaneous tasks (4\%, SD 10.0). Most participants reported seeing patients on a weekly basis $(89 \%, n=174)$ for 50 to 60 minutes $(96.9 \%, n=190)$. When asked how many patients are seen, full time workers $(n=154)$ reported an average of 17.06 weekly contacts ( $S D 4.60)$ with a range of 2.5- 26.5 cases. Participants were asked to rate how frequently they used key CBT procedures/methods in their therapy sessions on the 5-point Likert scale.

Most reported using some CBT components in most or every session. These were homework setting $(96 \%, n=187)$, homework review $(95 \%, n=186)$, and agenda setting $(90 \%, n=176)$. They were followed by referring to a formulation $(88 \%, n=173)$, applying 
an active CBT intervention $(79 \%, n=155)$, and thought evaluation $(68 \%, n=134)$. Only one person reported never setting an agenda.

\section{Behavioural experiments}

In CBT for depression and anxiety disorders, behavioural experiments are a key component of treatment. Thirty-one percent of respondents $(n=60)$ reported that they used behavioural experiments in most or every therapy session, 56\% $(n=110)$ reported using them in some sessions and $12 \%(n=23)$ using them in a few sessions. Almost everyone therefore used behavioural experiments as part of their clinical work. Therapist accompanied out-of-office experiments or exposure work were less frequently used with only $8.1 \%(n=16)$ of former trainees reporting using them in most or every session, $24 \%(n=46)$ using them in some sessions and $60 \%$ ( $n=118)$ using them in a few sessions. Session time restrictions and service policies/obstacles were often mentioned as reasons for not doing out-of-office behavioural experiments.

\section{Recording therapy sessions}

During training, trainees are encouraged to record all therapy sessions for personal reflection, planning future sessions, and discussion in supervision. On graduation, trainees were encouraged to continue to utilize therapy recordings in supervision meetings. A substantial change in therapy practice from the course was that only $26 \%(n=50)$ recorded therapy most or every session, with the majority of participants $(40 \%, n=77)$ reporting recording few therapy sessions for supervision purposes. No-one reviewed clips of live recordings for every session, only 4\% $(n=8)$ reviewed most sessions, with the majority $61 \%(n=118)$ reviewing few sessions. Seven percent $(n=13)$ reported they never reviewed sessions. In training and during routine clinical work, trainees use mandatory IAPT clinical outcome measures in 
every session (Kroenke, Spitzer and Williams, 2001; Spitzer, Kroenke, Williams and Löwe, 2006). With anxiety disorder cases, they are also encouraged to use disorder-specific outcome measures to inform their therapy. Sixty-seven percent $(n=131)$ reported using the disorderspecific outcome measures in most sessions.

\section{Evidence-based treatment}

The IAPT CBT training content is based on the HI National Curriculum (Department of Health, 2011). This curriculum incorporates NICE guidance evidence-based interventions for depression and anxiety disorders (www.nice.org.uk), and the Roth and Pilling CBT Competence Framework (2008). The follow-up survey focused on key active strategies across disorders embedded in the Competence Framework (Roth and Pilling, 2008), which had been taught and assessed on the training course, and asked about current adherence. Using the 5-point Likert scale, participants reported the following adherence in their posttraining clinical practice (Table 3).

Similar to the reporting on the general clinical strategies, therapist-accompanied outof-office experiments, PTSD site visits and OCD home visits showed lower adherence.

\section{[Insert Table 3 about here]}

\section{Number of sessions}

Trainees on the CBT training are encouraged to treat patients for the required number of therapy sessions as indicated in NICE guidance (www.nice.org.uk). Survey participants were asked to rate the average number of sessions their patients received (Table 4). Responses indicated that very few past trainees routinely provided grossly inadequate number of 
sessions (six or fewer) and there was some evidence that the numbers of sessions offered was greater in complex cases and personality disorders, as one would expect.

[Insert Table 4 about here]

\section{Complexity}

IAPT trainees are encouraged to take on suitable training cases in order to facilitate the learning of new skills and interventions across different presentations. Suitable training cases are defined as patients with clear presenting problems incorporating Axis 1 diagnoses and comorbidity. Exclusion criteria encompass an Axis 2 diagnosis, previous treatment resistant difficulties, active suicidality, and psychosis. Complexity ratings $(n=193)$ of past trainees' current cases indicated that they are still mainly treating Axis 1 diagnoses with co-morbidity $96 \%(n=186)$. They also reported more complexity in their cases posttraining, with $51 \%(n$ =99) reporting cases with Axis 2 disorders, $83 \%(n=160)$ cases with social/housing/unemployment difficulties, and 56\% $(n=109)$ cases presenting with treatment resistance. This is a trajectory we would expect posttraining.

\section{Adherence to NICE guidelines}

The majority of participants reported adherence to protocols, such as NICE guidance and Roth and Pilling's Competency Framework, most to all of the time $(66 \%, n=130)$. The primary reasons given for drifting from these protocols were patient complexity $(81 \%, n=$ $159)$ and service demands $(65 \%, n=128)$.

The main reasons given for complying with the protocols were associated with being satisfied with CBT or NICE guidelines $(67 \%, n=132)$, their straight-forward application $(65 \%, n=128)$, and frequent supervision $(38 \%, n=74)$. 


\section{CBT supervision}

During training, IAPT trainees receive weekly group supervision (groups of 3 for 90 minutes) from the CBT training course for their training cases. They also receive weekly CBT individual supervision for 1 hour in their IAPT Service for their other CBT cases. All clinical supervisors are BABCP-accredited CBT therapists. Course group supervision provides "close" (audio or video) supervision of CBT training cases with regular review of live therapy clips, reviewing whole therapy sessions with the use of competency measures (such as the CTS-R, Blackburn et al., 2001). Course supervision also encourages the use of outcome measures to inform therapy decisions. IAPT supervision guidance sets out an expectation for weekly outcome-focused CBT clinical supervision. In the follow-up survey, $89 \%(n=175)$ of participants reported receiving individual supervision, $8 \%(n=16)$ attended group and 3\% $(n=5)$ attended peer supervision. Supervision was received either weekly $(44 \%, n=86)$ or fortnightly $(39 \%, n=77)$ in the majority of cases and lasted 1 hour $(84 \%, n$ $=164)$. Former trainees were supervised in most instances by a clinical psychologist $(46 \%, n$ $=90)$, BABCP-accredited CBT therapist $(31 \%, n=61)$ or counselling psychologist $(16 \%, n=$ 32).

Trainees were asked how frequently their supervision focused on some of the key IAPT principles for supervision provision. Participants reported that the content of supervision mainly focused on case discussion $(76 \%, n=151)$ in every or most supervision sessions. Live assessment (either in vivo, video or audio), a major focus of all IAPT course supervision, was only used by a small number of ex-trainees in most or every supervision session ( $3 \%, n=6$ respondents). The majority of respondents reported using live assessment in few sessions $(60 \%, n=117)$ whilst $19 \%(n=36)$ reported never using live assessment in supervision. The discussion of disorder-specific outcome measures $(23 \%, n=45)$, role plays 
$(4 \%, n=6)$, and competency measures (like the CTS-R; $1 \%, n=2)$ were covered by few extrainees for most or every supervision session and followed a similar trend to using live assessments.

\section{CBT supervisors}

In terms of possible dissemination of CBT skills and practice, it was encouraging to see that $61 \%$ participants $(n=119)$ reported providing CBT supervision to other professionals. Again, supervision was primarily weekly $(77 \%, n=92)$, individual $(87 \%, n=104)$, and lasted 1 hour $(75 \%, n=89)$. The main recipient groups of this supervision were psychological well being practitioners/assistant psychologists $(38 \%, n=45)$, followed by trainee psychologists/trainee CBT therapists $(28 \%, n=33)$, clinical/counselling/health psychologists $(13 \%, n=15)$, and CBT therapists $(11 \%, n=13)$. A similar picture emerged regarding the content of supervision provided, with most participants stating case discussion is covered in most to every session $(91 \%, n=107)$, outcome measures reviewed $(41 \%, n=48)$, and live assessment $(9 \%, n=$ $10)$.

\section{Self-rated therapy competence}

Participants were asked to rate their CBT competence using the 6-point scale of the Dreyfus Model of Competence (Dreyfus and Dreyfus, 1980), a scale embedded within the Cognitive Therapy Rating Scale (Blackburn et al., 2001), which is used to assess CBT competence across UK IAPT CBT courses. The six levels of competence are: Incompetent, Novice, Advanced beginner, Competent, Proficient, and Expert.

Former trainees were asked to rate their competence retrospectively, before and after training, and to rate their current level of competence. Participants self-reported an improvement in competence across the time-points (Table 5 and Figure 2). 
[Insert Table 5/Figure 2 about here]

\section{CBT high intensity experience}

Survey participants were asked to fill in free text boxes about positive and negative aspects of their current clinical work. The following three themes emerged.

(1) Participants reflected on the value of the CBT training and the value of CBT. They reported enjoying their CBT work and liking a varied caseload. They also reported enjoying seeing patients improve and working in supportive work environments.

(2) The most common aspect they would prefer to change was the pressure of targets, high caseloads and a lack of time to reflect on clinical work.

(3) Participants also noted an increase of complex cases in their caseload since leaving training.

\section{Discussion}

The follow-up survey had a very high response rate $(92.5 \%)$ and many aspects of the findings are encouraging.

\section{Retention}

The survey indicates a high level of IAPT High Intensity (HI) workforce retention. The majority of IAPT trainees go on to work in IAPT services and stay there - a key finding. All HI trainees initially worked in IAPT services posttraining, and those who had moved on are 
mainly still in NHS posts delivering CBT in specialist services. The survey also indicated good career progression with trainees taking on supervision responsibilities.

\section{Clinical practice}

The majority of past trainees were still practising CBT, with 94\% stating that CBT remains their main therapy modality. Participants reported still enjoying their CBT work and evidenced good adherence to CBT principles and techniques taught on the course with the use of active CBT session work.

Ninety-five percent of therapists reported using activity scheduling and working on thoughts for depression, $89 \%$ reported using attention training for social phobia, and $81 \%$ reported still using behavioural experiments across anxiety disorders.

Few survey participants indicated that their clients routinely received a grossly inadequate number of sessions, with almost all participants indicating that they routinely provided either 7-12 sessions or 13-20 sessions, with the latter category being more common for complex cases. This is an important finding as recent studies have indicated that higher numbers of treatment sessions are associated with better clinical recovery (Gyani, Shafran, Layard and Clark, 2011; Vaillancourt, Manley and McNulty, 2015). The $3^{\text {rd }}$ Annual IAPT Report (HSCIC, 2015) paints a less rosy picture, with the national average for CBT sessions being 5.8. This suggests that IoPPN course trainees may be providing more sessions than the average IAPT HI therapist, perhaps because the course strongly emphasizes the importance of an adequate dose of therapy. Survey participants also reported improvement in their CBT competence posttraining. A high percentage of participants have also attained BABCP CBT therapist accreditation status (provisional or full).

There were, however, three key areas where trainees' reports of their posttraining experience were a cause for concern. 
(1) Less than a third of therapists reported doing out-of-office work with patients. This is a key component of NICE recommended psychological therapy, particularly in anxiety disorders, with out-of-office experiments, PTSD site visits, and home visits in OCD. Lack of adherence could reflect the way services are organized, but will impact negatively on therapy outcome.

(2) Although the respondents reported receiving regular supervision in their postqualification work, it was alarming that less than a quarter discussed outcome measures in supervision sessions. The IAPT model specifies that all clinicians (not just trainees) should have outcome-focused weekly supervision for their cases. This is again likely to have a negative impact on therapy and recovery rates as it means that supervision is less focused on what is needed to help recovery.

(3) Recording and reflecting on therapy and the use of "live" supervision, a key focus of CBT training and course supervision, and a BABCP accreditation requirement, was being used by few trainees.

Lack of close supervision can also have an adverse effect on patient improvement (Lambert, Harmon, Slade, Whipple and Hawkins, 2005).

\section{Study limitations}

There are a number of limitations to this research that affect the interpretation of these results. The survey was conducted on IoPPN/KCL past trainees only, who are working predominantly in London. The training institution and past trainers conducted the survey and reviewed responses. The course attracts a higher percentage of clinical and counselling psychologists compared to other HI courses nationally, and $79 \%$ of trainees had attained a $1^{\text {st }}$ 
class or 2:1 undergraduate degree. We cannot necessarily assume that our findings will generalize to IAPT training courses as a whole.

The analyses were conducted on the basis of self-report data, which may carry inherent biases. There is evidence to indicate that therapists may be poor assessors of their own therapy skills (Brosan, Reynolds and Moore, 2008; Lambert et al., 2005). Judgements of the complexity of their caseloads are also subjective. During training, trainees are taught diagnostic assessment of Axis 1 anxiety disorders and depression. However, they do not have formal training in diagnosing personality disorders. The current reliability of their diagnostic judgements is also unknown.

Finally, some of the raw data are skewed owing to the fact that the follow-up data for years 1-5 was collected in 2014 when Year 1 were 5 years post CBT training and Year 5 had finished only 1 year ago. Year 6 was collected in September 2015.

\section{Conclusions}

This survey provides some indication that staff retention and skills transfer to the workplace is enhanced by a national directive setting out standards and implementation guidelines (www.iapt.nhs.uk). IAPT trainees are employed full-time as CBT therapists in IAPT services after training, and as indicated in this survey, a high percentage of their time continues to be spent delivering CBT to patients they have been trained to treat. The IAPT trainees also continue to receive on-going clinical supervision. A large number of past trainees are now supervising current trainees or junior CBT staff, which helps embed CBT skills learnt during training into the workplace. This survey also highlighted a number of shortfalls in the transference of evidence-based practise from training to routine delivery which, if rectified by IAPT services, could enhance therapy competence and aid patient recovery. Further research 
that follows up IAPT CBT trainees to assess CBT therapy skill and clinical outcome posttraining is required.

\section{Acknowledgements}

With thanks to Suzanne Byrne, Nick Grey and Colette Hirsch for their invaluable comments on the manuscript, and with many thanks to all the past IAPT CBT trainees who participated in this Survey.

Financial support: This research received no specific grant from any funding agency, commercial or not-for-profit sectors.

Conflict of interests: Sheena Liness is director of the IAPT High Intensity therapies training course that is the subject of this report. David M. Clark is NHS England's Clinical Advisor for the IAPT programme. Susan Lea, Steffen Nestler and Hannah Parker have no conflict of interest with respect to this publication.

\section{References}

Ashworth, P., Williams, C. and Blackburn, I. M. (1999). What becomes of cognitive therapy trainees? A survey of trainees' opinions and current clinical practice after postgraduate cognitive therapy training. Behavioural and Cognitive Psychotherapy, 27, 267-277.

Blackburn, I.-M., James, I. A., Milne, D. L., Baker, C., Standart, S., Garland, A., et al. (2001). The revised Cognitive Therapy Scale (CTS-R): psychometric properties. Behavioural and Cognitive Psychotherapy, 29, 431-446 
Brooker, C. and Brown, M. (1986). National follow-up survey of practising nurse therapists. In D. Cormack and W. Reynolds (Eds.), Psychiatric and Mental Health Nursing: theory and practice (1 st edn.). Dordrecht: Springer Science+Business Media.

Brooker, C., Saul, C., Robinson, J., King, J. and Dudley, M. (2003). Is training in psychosocial interventions worthwhile? Report of a psychosocial intervention trainee follow-up study. International Journal of Nursing Studies, 40, 731-747.

Brosan, L., Reynolds, S. and Moore, R. G. (2008). Self-evaluation of cognitive therapy performance: do therapists know how competent they are? Behavioural and Cognitive Psychotherapy, 36, 581.

Department of Health (2008). IAPT Implementation Plan: national guidelines for regional delivery. London: The Stationary Office. Available at http://www.iapt.nhs.uk/silo/files/implementation-plan-national-guidelines-forregional-delivery.pdf.

Department of Health (2011). National curriculum for high intensity cognitive behavioural therapy courses, UK. The Stationery Office. Available at: http://iapt.nhs.uk/silo/files/national-curriculum-for-high-intensity-cognitivebehavioural-therapy-courses.pdf.

Dreyfus, S. E. and Dreyfus, H. L. (1980). A Five-Stage Model of the Mental Activities Involved in Directed Skill Acquisition: DTIC Document.

Fadden, G. (1997). Implementation of family interventions in routine clinical practice following staff training programs: a major cause for concern. Journal of Mental Health, 6, 599-612. 
Gournay, K., Denford, L., Parr, A.-M. and Newell, R. (2000). British nurses in behavioural psychotherapy: a 25-year follow-up. Journal of Advanced Nursing, 32, 343-351.

Gyani, A., Shafran, R., Layard, R. and Clark, D. M. (2011). Enhancing Recovery Rates in IAPT Services: lessons from analysis of the Year One data. London: Improving Access to Psychological Therapies.

Herschell, A. D., Kolko, D. J., Baumann, B. L. and Davis, A. C. (2010). The role of therapist training in the implementation of psychosocial treatments: a review and critique with recommendations. Clinical Psychology Review, 30, 448-466.

Health and Social Care Information Centre (2015). Psychological Therapies: annual report on the use of IAPT services; England 2014/15. Leeds: Community and Mental Health team, HSCIC.

IAPT Programme NHS England (2015). 2014 Adult IAPT Workforce Census Report. Retrieved from http://www.iapt.nhs.uk/silo/files/2014-adult-iapt-workforce-censusreport.pdf

\section{Kavanagh, D. J., Piatowska, O., Clark, D., O'Halloran, P., Manicavasagar, V., Rosen,} A., et al. (1993). Application of cognitive-behavioural family intervention for schizophrenia in multidisciplinary teams: what can the matter be? Australian Psychologist, 28, 181-188.

\section{Kennedy-Merrick, S. J., Haarhoff, B. A., Stenhouse, L. M., Merrick, P. L. and}

Kazantzis, N. (2008). Training cognitive behavioural therapy practitioners in New Zealand: from university to clinical practice. New Zealand Journal of Psychology, 37, 8-17. 
Kjøge, A., Turtumøygard, T., Berge, T. and Ogden, T. (2015). From training to practice: a survey study of clinical challenges in implementing cognitive behavioural therapy in Norway. The Cognitive Behaviour Therapist, 8(e16), 1-16.

Kroenke, K., Spitzer, R. L. and Williams, J. B. W. (2001). The PHQ-9. Journal of General Internal Medicine, 16, 606-613.

Lambert, M. J., Harmon, C., Slade, K., Whipple, J. L. and Hawkins, E. J. (2005). Providing feedback to psychotherapists on their patients' progress: clinical results and practice suggestions. Journal of Clinical Psychology 61, 165-174.

MacLiam, F. (2015). Cognitive behavioural psychotherapy graduates in Ireland: a follow-up survey of graduates from an Irish university. Irish Journal of Psychological Medicine, $32,187-195$.

Milne, D., Gorenski, O., Westerman, C., Leek, C. and Keengan, D. (2000). What does it take to transfer training? Psychiatric Rehabilitation Skills, 4, 259-281.

Newell, R., and Gournay, K. (1994). British nurses in behavioural psychotherapy: a 20-year follow-up. Journal of Advanced Nursing, 20, 53-60.

NHS (2015). Pay Circular - Agenda for Change 1/2015, UK: the NHS Staff Council.

Available at: http://www.nhsemployers.org/case-studies-and-resources/2015/05/paycircular-agenda-for-change-1-2015 .

Roth, A. D. and Pilling, S. (2008). Using an evidence-based methodology to identify the competences required to deliver effective cognitive and behavioural therapy for depression and anxiety disorders. Behavioural and Cognitive Psychotherapy, 36, 129147.

Ryan, D., Cullinan, V. and Quayle, E. (2005). A survey of trainees' opinions and current clinical practice after behaviour therapy training. Journal of Psychiatric and Mental Health Nursing, 12, 199-208. 
Spitzer, R. L., Kroenke, K., Williams, J. W. and Löwe, B. (2006). A brief measure for assessing generalized anxiety disorder: the GAD-7. Archives of Internal Medicine, $166,1092-1097$.

Vaillancourt, K., Manley, J. and McNulty, N. (2015). Why has our recovery rate dropped? An audit examining waiting times, starting scores and length of treatment in relation to recovery within an IAPT service. The Cognitive Behaviour Therapist, 8(e7), 1-14. 
Table 1. Demographic information of respondents $(N=196)$

\begin{tabular}{|c|c|c|c|}
\hline Characteristic & Categories & Count & Percentage \\
\hline \multirow[t]{2}{*}{ Response rate } & Responders & 196 & 92.5 \\
\hline & Non-responders & 16 & 7.5 \\
\hline \multirow[t]{4}{*}{ Age } & $20 s$ & 20 & 10.2 \\
\hline & $30 \mathrm{~s}$ & 130 & 66.3 \\
\hline & $40 \mathrm{~s}$ & 31 & 15.8 \\
\hline & $50 s$ & 15 & 7.7 \\
\hline \multirow[t]{2}{*}{ Gender } & Female & 161 & 82.1 \\
\hline & Male & 35 & 17.9 \\
\hline \multirow[t]{5}{*}{ Undergraduate degree } & $1^{\text {st }}$ (or equivalent) & 52 & 26.5 \\
\hline & $2: 1$ (or equivalent) & 104 & 53.1 \\
\hline & $2: 2$ (or equivalent) & 30 & 15.3 \\
\hline & $3^{\text {rd }}$ (or equivalent) & 3 & 1.5 \\
\hline & $n / a$ & 7 & 3.6 \\
\hline \multirow[t]{8}{*}{ Core professions } & Clinical psychologists & 71 & 36.2 \\
\hline & PWP's* & 58 & 29.6 \\
\hline & Counselling psychologists & 30 & 15.3 \\
\hline & Mental health nurses & 18 & 9.2 \\
\hline & Occupational therapists & 7 & 36 \\
\hline & Accredited counsellors / & 7 & 3.6 \\
\hline & Psychotherapists & 6 & 3.1 \\
\hline & Miscellaneous professions & 6 & 3.1 \\
\hline \multirow{5}{*}{$\begin{array}{l}\text { Qualification in } \\
\text { core profession }\end{array}$} & 2010s & 80 & 40.8 \\
\hline & $2000 s$ & 93 & 47.4 \\
\hline & $1990 \mathrm{~s}$ & 15 & 7.7 \\
\hline & $1980 \mathrm{~s}$ & 5 & 2.6 \\
\hline & $\mathrm{n} / \mathrm{a}$ & 3 & 1.5 \\
\hline \multirow[t]{5}{*}{ Ethnicity } & White British & 120 & 61.2 \\
\hline & White Other & 38 & 19.4 \\
\hline & Asian/Asian British & 20 & 10.2 \\
\hline & Black $^{* *}$ & 14 & 7.2 \\
\hline & Mixed & 4 & 2.0 \\
\hline
\end{tabular}

* Category includes Psychological Wellbeing Practitioners (PWP's) and Postgraduate Mental Health Workers. Professionals in this category did not have a clinical core profession. ${ }^{* *}$ Category includes Black, Black British, Black British African, Black British Caribbean, and Black African 
Figure 1. Retention of HI trainees in IAPT services across 6 cohorts (2008-2014)

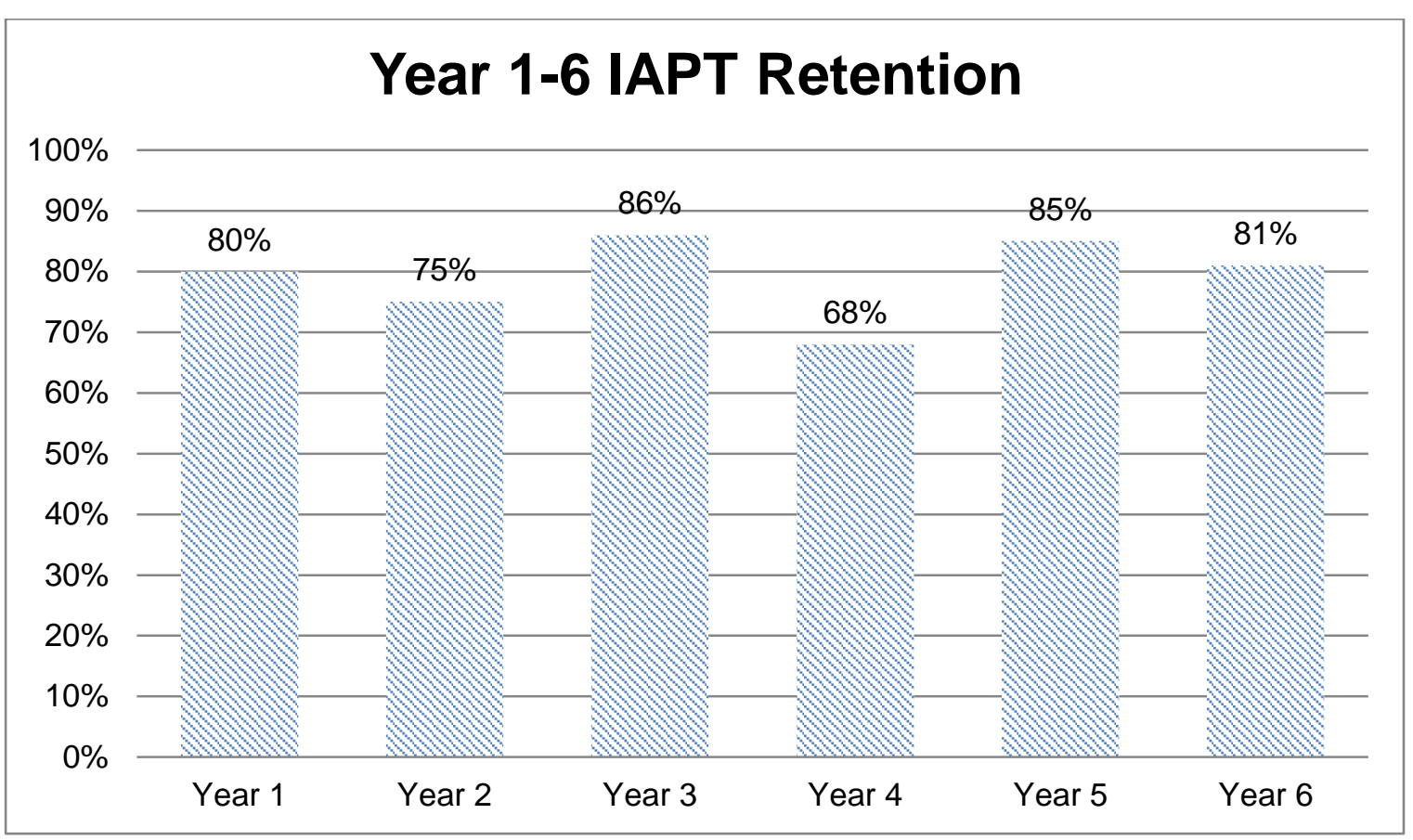


Table 2. Distribution of current work duties as reported by former trainees $(n=196)$

\begin{tabular}{lrr}
\hline Type of work & Mean percentage & Standard Deviation $(S D)$ \\
\hline Clinical work & 75.9 & 22.14 \\
Research & 1.7 & 3.91 \\
Managerial & 5.5 & 10.70 \\
Teaching/training others & 3.8 & 8.72 \\
Supervision (giving) & 9.5 & 10.00 \\
Other & 3.6 & 9.97 \\
\hline
\end{tabular}


Table 3. Reported prevalence of NICE-recommended CBT activities, split by disorder (of $n=196$ )

\begin{tabular}{|c|c|c|}
\hline Disorder & CBT activity & $\begin{array}{l}\text { \% responses for "most" and } \\
\text { "every" patient categories } \\
\text { (Number of respondents) }\end{array}$ \\
\hline \multirow[t]{2}{*}{ Depression } & Activity scheduling & $95(186)$ \\
\hline & Working with thoughts & $95(186)$ \\
\hline \multirow[t]{2}{*}{ Social phobia } & Attention focus training & 89 (175) \\
\hline & Video feedback & $79(155)$ \\
\hline \multirow[t]{3}{*}{ PTSD } & Imaginal reliving & $79(155)$ \\
\hline & Memory discrimination & $54(106)$ \\
\hline & Site visit & $24(47)$ \\
\hline \multirow{2}{*}{ OCD } & Exposure work & $72(141)$ \\
\hline & Home visit & $15(30)$ \\
\hline Panic Disorder & Behavioural experiments & $83(163)$ \\
\hline \multirow[t]{3}{*}{ Anxiety Disorders in General } & Dropping safety behaviours & $95(186)$ \\
\hline & In-office experiments & $81(159)$ \\
\hline & Out of office experiments & $30(59)$ \\
\hline
\end{tabular}


Table 4. Number of sessions received by clients as reported by former trainees, split by diagnosis $(n=193)$

\begin{tabular}{llll}
\hline $\begin{array}{l}\text { Number of received } \\
\text { sessions }\end{array}$ & Depression & Anxiety & $\begin{array}{l}\text { Complex/personality } \\
\text { disorders }\end{array}$ \\
\hline $1-6$ & $1 \%(2)$ & $1 \%(2)$ & $2 \%(4)$ \\
$7-12$ & $55 \%(107)$ & $76 \%(149)$ & $23 \%(45)$ \\
$13-20$ & $39 \%(76)$ & $18 \%(35)$ & $48 \%(94)$ \\
$21+$ & $2 \%(3)$ & $2 \%(4)$ & $9 \%(18)$ \\
\hline
\end{tabular}


Table 5. Former trainees' self-ratings of their CBT competence across three time points

\begin{tabular}{lrrr}
\hline & $\begin{array}{r}\text { Before } \\
\text { CBT }\end{array}$ & $\begin{array}{r}\text { After CBT } \\
\text { training }\end{array}$ & Now \\
& training & & \\
\hline Expert & $0.0 \%$ & $0.0 \%$ & $0.5 \%$ \\
Proficient & $0.0 \%$ & $13.3 \%$ & $42.9 \%$ \\
Competent & $7.1 \%$ & $61.7 \%$ & $52.0 \%$ \\
Advanced & $43.4 \%$ & $20.4 \%$ & $2.6 \%$ \\
beginner & $38.8 \%$ & $3.1 \%$ & $0.5 \%$ \\
Novice & $8.7 \%$ & $0.0 \%$ & $0.0 \%$ \\
Incompetent & $98.0 \%$ & $98.5 \%$ & $98.5 \%$ \\
\hline Interim total & & & \\
\hline
\end{tabular}


Figure 2. Former trainees' self-ratings of their CBT competence across three time points

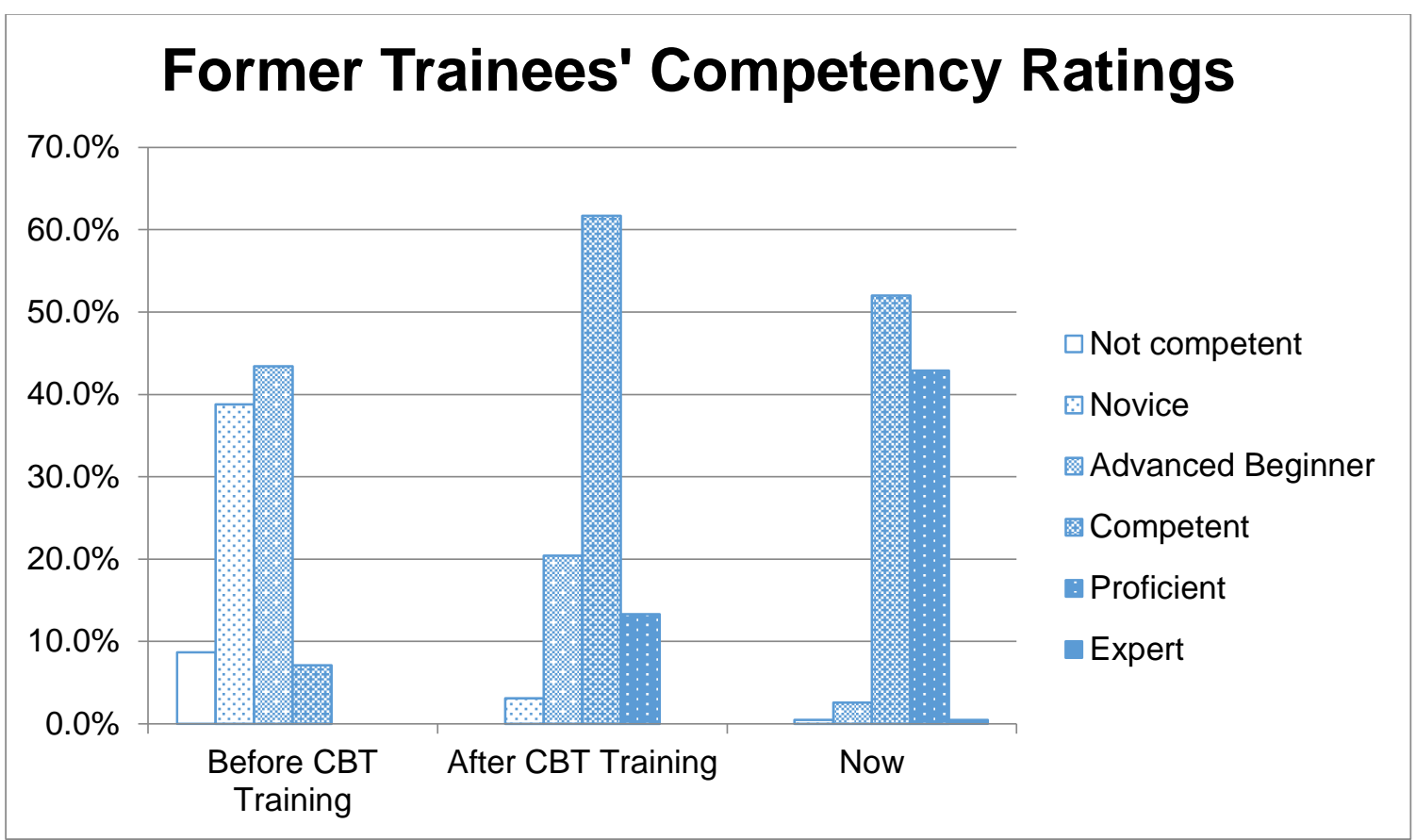

